\title{
Some Remarks on Trigonometric Interpolation on the $n$-Torus
}

\author{
W. SICKEL
}

Let $T^{n}$ be the $n$-torus and $\left(I_{j} f\right)_{j=0}^{\infty}$.

$$
l_{j} f(x)=\sum_{k_{1}=-j}^{j} \ldots \sum_{k_{n}=-j}^{j} f\left(\frac{2 \pi k}{2 j+1}\right) \prod_{j=1}^{n} \frac{\sin \left(\frac{2 j+1}{2}\left(x_{i}-\frac{2 \pi k_{j}}{2 j+\frac{1}{2}}\right)\right)}{(2 j+1) \sin \frac{1}{2}\left(x_{i}-\frac{2 \pi k_{i}}{2 j+1}\right)} \quad\left(\begin{array}{l}
x=\left(x_{1}, \ldots, x_{n}\right) \in \mathbf{R}^{n} \\
k=\left(k_{1}, \ldots, k_{n}\right)<\mathbf{z}^{n}
\end{array}\right)
$$

the sequence of Lagrange interpolating polynomials. Then we give a complete characterization of the set of functions $f$ with

$$
\left(\sum_{j=1}^{\infty}\left[j s\left\|_{f}-L_{j} f \mid L_{p}\left(\mathbf{T}^{n}\right)\right\|^{q}\right)^{1 / q}<\infty \quad \text { if } 1<p<\infty, 0<q \leq \infty, s>n / p\right.
$$

and

$$
\left.\| \sum_{j=1}^{\infty}\left[j^{s} \mid f(x)-I_{j} f(x)\right]^{q}\right)^{1 / q} \mid L_{p}\left(T^{n}\right) \|<\infty \quad \text { if } 1<p<\infty, 0<q \leq \infty, s<n / p
$$

in terms of Besov-Triebel-Lizorkin spaces on $T^{n}$.

Key words: Periodic spaces of Besov-Triebel-Lizorkin type, Lagrange interpolating polynomials, approximation of functions

AMS subject classification: $41 \mathrm{~A} 25,42 \mathrm{~A} 15,46 \mathrm{E} 15$

\section{Introduction}

As usual, $R^{n}$ denotes the Euclidean $n$-space, $Z^{n}$ the set of all lattice points having integer components, $N$ the set of all natural numbers and $N_{0}$ the set of all non-negative integers. The aim of the paper is to show that the Besov-Triebel-Lizorkin spaces on the $n$-torus $\mathbf{T}^{n}$ can be completely characterized by the sequence $\left(I_{j} f\right)_{j=0}^{\infty}$ of Lagrange interpolating polynomials. Here $I_{j} f$ is given by

$$
\sum_{k_{1}=-j}^{j} \ldots \sum_{k_{n}=-j}^{j} f\left(\frac{2 \pi \mathrm{k}}{2 j+1}\right) \prod_{j=1}^{n} \frac{\sin \left(\frac{2 j+1}{2} x_{i}-k_{i} \pi\right)}{(2 j+1) \sin \frac{1}{2}\left(x_{i}-\frac{2 \pi k_{i}}{2 j+1}\right)}, \begin{aligned}
& x=\left(x_{1}, \ldots, x_{n}\right) \in \mathbf{T}^{n} \\
& k=\left(k_{1}, \ldots, k_{n}\right) \in \mathbf{Z}^{n}
\end{aligned} .
$$

It turns out that for periodic continuous functions $f$ the following equivalences are true $(1<p<\infty, s>n / p)$ :

$$
\begin{array}{ll}
f \in B_{p, q}^{s}\left(\mathbf{T}^{n}\right) \Leftrightarrow\left(\sum_{j=1}^{\infty}\left[j s-1 / q \| f-I_{j} f\left|L_{p}\left(\mathbf{T}^{n}\right)\right|\right]^{q}\right)^{1 / q}<\infty . & (0<q \leq \infty), \\
f \in F_{p, q}^{s}\left(\mathbf{T}^{n}\right) \Leftrightarrow\left\|\left(\sum_{j=1}^{\infty}\left[j^{s-1 / q}\left|f(x)-I_{j} f(x)\right|\right]^{q}\right)^{1 / q} \mid L_{p}\left(\mathbf{T}^{n}\right)\right\|<\infty \quad(1<q<\infty) .
\end{array}
$$

The main tools of proof used here are the characterization of the underlying function spa- 
ces via approximation and the $L_{p}$-stability of trigonometric polynomials $t$ of degree $t \leq j$ expressed by the following inequalities:

$c_{1}\left(\frac{1}{(2 j+1)^{n}} \sum_{k_{1}=-j}^{j} \ldots \sum_{k_{n}=-j}^{j}\left|t\left(\frac{2 \pi k}{2 j+1}\right)\right| p\right)^{1 / p} \leq\left\|\left.t\left|L_{p}\left(\mathrm{~T}^{n}\right) \| \leq c_{2} \sum_{k_{1}=-j}^{j} \ldots \sum_{k_{n}=-j}^{j}\right| t\left(\frac{2 \pi k}{2 j+1}\right)\right|^{p}\right)^{1 / p}(0.2)$

$(1<p<\infty)$ for some constants $c_{1}, c_{2}>0$, independent of $t$ and $j$ (cf. A. Zygmund [19], P.1.

Lizorkin and D.G. Orlovskij [4]).

The paper is organized as follows:

After collecting some necessary informations about Besov-Triebel-Lizorkin spaces on $\mathrm{T}^{n}$ in the first section, Section 2 deals with our main result concerning the characterization of the function spaces. Therefore we investigate the uniform boudedness of $l_{j}$ in $\left\|\cdot \mid L_{p}\left(T^{n}\right)\right\|, 1<p<\infty$. As a complement and more or less to show the great similarity between approximation via partial sums and approximation via Lagrange interpolating polynomials the aliasing error $f-I_{j} f$ is also treated in $\left\|\cdot \mid C\left(T^{n}\right)\right\|$. Finally, in Section 3 we deal with approximation in stronger norms than $\left\|\cdot \mid L_{p}\left(T^{n}\right)\right\|$, for instance in $\left\|\cdot \mid W_{p}^{m}\left(T^{n}\right)\right\|$.

\section{Besov-Triebel-Lizorkin spaces}

1.1 Notations and definitions. The $n$-torus $T^{n}$ may be represented by the set

$$
\left\{x \in \mathbb{R}^{n}:-\pi \leq x_{j} \leq \pi(j=1, \ldots, n)\right\},
$$

where opposite sides are identified. $D_{\pi}$ and $D_{\pi}$ denote the set of all complex-valued infinitely differentiable functions on $\mathrm{T}^{n}$ and its dual space, respectively. Furthermore we put

$$
\hat{f}(k)=(2 \pi)^{-n} f\left(e^{-i k x}\right) \quad\left(k \in Z^{n}, f \in D_{\pi}^{*}\right) \text {. }
$$

Then any $f \in D_{\pi}^{\prime}$ can be represented by its Fourier series

$$
f=\sum_{k \in \mathbf{Z}^{n}} \hat{f}(k) \mathrm{e}^{\mathrm{i} k x} \text { (convergence in } D_{\pi}^{\prime} \text { ) }
$$

(cf. H.-J. Schmeißer and H. Triebel [14]). The space of continuous functions on $T^{n}$ is denoted by $C\left(\mathbf{T}^{\prime \prime}\right)$, the space of $p$-th power integrable functions by $L_{p}\left(T^{n}\right)$. If there is no confusion possible we drop $T^{n}$ in notations.

Let $\psi$ be an infinitely differentiable function with the properties

$$
\psi(x)=1 \text { if }|x| \leq 1 \text { and } \psi(x)=0 \text { if }|x| \geq 3 / 2
$$

Further we put

$$
\varphi_{0}(x)=\psi(x), \varphi_{1}(x)=\psi(x / 2)-\psi(x), \varphi_{l}(x)=\varphi_{1}\left(2^{-1+1} x\right) \quad(1=2,3, \ldots) .
$$

Hence, we have

$$
\sum_{l=0}^{\infty} \varphi_{1}(x)=1\left(x \in \mathbb{R}^{n}\right)
$$

Definition: Let $0<q \leq \infty$ and $-\infty<s<\infty$.

(i) Let $1 \leq p \leq \infty$. Then

$$
B_{p, q}^{s}\left(\mathrm{~T}^{n}\right)=\left\{f \in D_{\pi}^{*}:\left\|f \mid B_{p, q}^{s}\right\|=\left(\sum_{i=0}^{\infty} 2^{1 s q}\left\|\sum_{k \in Z^{n}} \varphi_{l}(k) \hat{f}(k) \mathrm{e}^{i k x} \mid L_{p}\right\|^{q}\right)^{1 / q}<\infty\right\} .
$$


(ii) Let $1<p<\infty$. Then

$$
F_{p, q}^{s}\left(\mathbf{T}^{n}\right)=\left\{f \in D_{\pi}^{\prime}:\left\|f\left|F_{p, q}^{s}\|=\|\left(\sum_{i=0}^{\infty} 2^{l s q}\left|\sum_{k \in Z^{n}} \varphi_{l}(k) \hat{f}(k) e^{i k x}\right|^{q}\right)^{1 / q}\right| L_{p}\right\|<\infty\right\} .
$$

Remark 1: All spaces defined above are quasi-Banach spaces (Banach spaces if $q \geq 1$ ). They are independent of the special choice of $\psi$ in (1.1) (equivalent quasi-norms). These periodic spaces of Besov-Triebel-Lizorkin type are extensively investigated in the book by H.-J. Schmeißer and H. Triebel [14].

Remark 2: The above definition can be understand as a uniform approach to different types of classical function spaces. In particular, we have

(i) $F_{p, 2}^{0}=L_{p}$,

(ii) $F_{p, 2}^{m}=W_{p}^{m}$ (Sobolev spaces) if $m \in \mathbb{N}$,

(iii) $B_{p, q}^{s}=\Lambda_{p, q}^{s}$ (Besov-Lipschitz classes) if $s>0$, and

(iv) $B_{\infty, \infty}^{s}=C^{s}$ (Hoelder-Zygmund classes) if $s>0$

(cf. H. -J. Schmeißer and H. Triebel [14]).

Remark 3: Of some importance are the embedding relations

$$
\left(B_{p, q}^{s} \cup F_{p, q}^{s}\right) \subset \rightarrow L_{p} \text { if } s>0
$$

and

$$
\left(B_{p, q}^{s} \cup F_{p, q}^{s}\right) \subset \rightarrow C \text { if } s>n / p
$$

(cf. H. -J. Schmeißer and H. Triebel [14]).

1.2 Characterization via approximation. The spaces defined above are well-adapted to problems in approximation theory. To show this we recall the following facts. Let

$$
T_{j}=\left\{t \in D_{\pi}^{;}: \hat{t}(k)=\text { o for all } k \in Z^{n},|k|>j\right\} \quad\left(j \in \mathbf{N}_{0}\right) .
$$

Let $X$ be an appropriate quasi-Banach space. Then we put

$$
E_{j}(f, X)=\inf _{g \in T_{j}}\|f-g \mid X\|\left(j \in \mathbb{N}_{o}\right) .
$$

Proposition 1: Let $0<q \leq \infty$.

(i) Let $1 \leq \mathrm{p} s \infty$ and $s>0$. Then

$$
B_{p, q}^{s}=\left\{f \in L_{p}:\left\|f \mid L_{p}\right\|+\left(\sum_{i=0}^{\infty}\left[(1+j)^{s-1 / q} E_{j}\left(f, L_{p}\right]^{q}\right)^{1 / q}<\infty\right\}\right.
$$

in the sense of equivalent quasi-norms.

(ii) Let

$$
S_{j} f(x)=\sum_{\left|k_{1}\right| \leq j} \ldots \sum_{\left|k_{n}\right| \leq j} \hat{f}(k) \mathrm{e}^{\mathrm{i} k x} \quad\left(k=\left(k_{1}, \ldots, k_{n}\right), j \in \mathbb{N}_{0}\right) .
$$

We can replace $E_{j}\left(f, L_{p}\right)$ by $\left\|f-S_{j} f \mid L_{p}\right\|$ in (i) if $1<p<\infty$. 
(iii) Let

$$
V_{j} f(x)=\sum_{k \in \mathbf{Z}^{n}} \psi\left(j^{-1} k\right) \hat{f}(k) \mathrm{e}^{i k x} \quad(j \in \mathrm{N}),
$$

where $\psi$ is the function from (1.1). Then $E_{j}\left(f, L_{p}\right)$ can be replaced by $\left\|f-V_{j} f \mid L_{p}\right\|$ in (i).

Proposition 2: Let $1<p, q<\infty$ and $s>0$. Then

$$
F_{p, q}^{s}=\left\{\begin{array}{ll} 
& 3\left\{g_{j}\right\}_{j=1}^{\infty}, g_{j} \in T_{j} \text { for } j \in \mathrm{N}, \text { such that } g_{j} \rightarrow f \text { in } L_{p} \text { and } \\
& \left\|g_{1}\left|L_{p}\|+\|\left(\sum_{j=1}^{\infty}\left[j^{s-1 / q}\left|f(x)-g_{j}(x)\right|\right]^{q}\right)^{1 / q}\right| L_{p}\right\|<\infty
\end{array}\right\}
$$

in the sense of equivalent norms. Moreover, we can choose $g_{j}=S_{j} f(j \in \mathbb{N})$.

Remark 4: Proofs of Propositions 1 and 2 may be found in H.-J. Schmeißer and H. Triebel [14] and W. Sickel [15] (cf. also H.-J. Schmeißer and W. Sickel [12, 13]).

Remark 5: For later use we mention also that $(0<q<\infty, s \geq 0)$

$$
\left\|f-S_{j} f \mid B_{p, q}^{s}\right\| \underset{j \rightarrow \infty}{\longrightarrow} 0 \text { if } 1<\rho<\infty \text { and }\left\|f-V_{j} f \mid B_{p, q}^{s}\right\| \underset{j \rightarrow \infty}{\longrightarrow} 0 \text { if } 1 \leq p \leq \infty \text {. }
$$

These are consequences of

$$
\hat{f}(k)-\widehat{S_{j} f}(k)=0 \text { if }\left|k_{j}\right|<j(i=1, \ldots, n) \text { and } \hat{f}(k)-\widehat{V_{j} f}(k)=0 \text { if }|k|<j
$$

and of

$$
\sup _{j}\left\|S_{j} f\left|L_{p}\|\leq c\| f\right| L_{p}\right\|(1<p<\infty) \text { and } \sup _{j}\left\|V_{j} f\left|L_{p}\|\leq c\| f\right| L_{p}\right\|(1 \leq p \leq \infty) .
$$

\section{Trigonometric interpolation}

We start with a uniform lattice on $\mathbf{T}^{n}$, characterized by the nodes

$$
x^{r}=\left(x_{1}^{r}, \ldots, x_{n}^{r}\right)=\left(\frac{2 \pi r_{1}}{2 j+1}, \ldots, \frac{2 \pi r_{n}}{2 j+1}\right)\left(-j \leq r_{i} \leq j(i=1, \ldots, n), j \in \mathbf{N}_{0}\right), r \in \mathbb{Z}^{n} .
$$

Let

$$
Q_{m}^{j}=\left\{k \in Z^{n}:-j-1 / 2 \leq k_{j}-m_{i}(2 j+1) \leq j+1 / 2(j=1, \ldots, n)\right\} \quad\left(m \in \mathbf{Z}^{n}, j \in \mathbb{N}_{0}\right)
$$

and $f \in C$. Then the function $I_{j} f$ defined by $(0.1)$ is the unique solution of

$$
g\left(x^{r}\right)=f\left(x^{r}\right), r \in Q_{0}^{j} \text { and } \hat{g}(k)=0 \text { if } k, Q_{0}^{j} .
$$

Suppose additionally

$$
\sum_{k \in Z^{n}}|\hat{f}(k)|<\infty .
$$

Then we can rewrite

$$
I_{j} f(x)=\sum_{m \in \mathbb{Z}^{n}}\left(\sum_{k \in Q_{m}^{j}} \hat{f}(k) \mathrm{e}^{i k x}\right) \mathrm{e}^{-i m(2 j+1) x} \quad\left(j \in \mathbb{N}_{0}\right)
$$

( cf. A. Zygmund [19]). 
Remark 6: Formula (2.2) shows the great similarity between Lagrange polanomials and Whittaker's cardinal series. The latter one is defined as

$$
I_{j}^{*} f(x)=\sum_{k \in \mathbf{Z}^{n}} f\left(\frac{2 \pi k}{2 j+1}\right) \prod_{i=1}^{n} \frac{\sin \left(\frac{2 j+1}{2} x_{i}-k_{i} \pi\right)}{\left(\frac{2 j+1}{2} x_{i}-k_{i} \pi\right)} \quad\left(x \in \mathbf{R}^{n}\right) .
$$

We have the identity

$$
I_{j}^{*} f(x)=\sum_{k \in Z^{n}}\left(F^{-1}\left[x_{m}^{j} F f\right]\right)(x) \mathrm{e}^{\cdot \mathrm{i} m(2 j+1) x},
$$

where $F, F^{-1}$ are the Fourier transform and its inverse, respectively, and $\chi_{m}^{j}$ denotes the characteristic function of $Q_{m}^{j}$ (cf. P.L. Butzer [3], W. Sickel [16]).

Remark 7: If we put $A_{\pi}=\left\{f \in D_{\pi}^{\prime}: \sum_{k \in Z^{n}}|\hat{f}(k)|<\infty\right\}$, then $B_{2,1}^{n / 2} \hookrightarrow \rightarrow A_{\pi} \hookrightarrow \rightarrow B_{\infty, 1}^{o}$ (cf. H. Triebel [17]).

In our investigations a crucial role is played by the following

Lemma 1: Let $1<p<\infty$.

(i) There exists a constant $c$ such that

$$
\left\|I_{j} f\left|L_{p}\left\|\leq c(1+j)^{-n / p}\right\| f\right| B_{p, 1}^{n / p}\right\|\left(j \in \mathrm{N}_{0}\right)
$$

holds for all $f \in B_{p, 1}^{n / p}$ with $\hat{f}(k)=0, k \in Q_{0}^{j}$.

(ii) There exists a constant $c$ such that

$$
\left\|f-I_{j} f\left|L_{p}\left\|\leq c(1+j)^{-n / p}\right\| f\right| B_{p, 1}^{n / p}\right\|\left(j \in N_{0}\right)
$$

holds for all $f \in B_{p, 1}^{n / p}$.

Proof: First, note that $I_{j}$ is a projection, that means $I_{j} f=f$ for all $f$ with $\hat{f}(k)=0$, $k \in Q_{0}^{j}$. Now we split

$$
f-I_{j} f=f-S_{j} f+I_{j}\left(S_{j} f-f\right) .
$$

To prove (2.4) we can use Proposition 1 and (2.3). So, it remains to prove (2.3). Let $\left\{\varphi_{l}\right\}$ be the system defined in (1.2). We put $f_{l}(x)=\sum_{k \in Z^{n}} \varphi_{l}(k) \hat{f}(k) \mathrm{e}^{i k x}\left(1 \in \mathbf{N}_{0}\right)$. Let $2^{t} s j s$ $2^{t+1}$. The properties of $\Psi$ guarantee $I_{j}\left(S_{j} f-f\right)=\sum_{l=t-1}^{\infty} I_{j}\left(S_{j} f_{l}-f_{l}\right)$ in $D_{\pi}^{\prime}$. Applying (0.2), using the interpolation property of $I_{j} f_{1} I_{j} f(k)=0$ if $k \Leftrightarrow Q_{0}^{j}$ and $\hat{f}_{l}(k)=0$ if $|k|>(3 / 2) 2^{1-1}$ we find

$$
\begin{aligned}
\left\|J_{j}\left(S_{j} f_{l}-f_{l}\right) \mid L_{p}\right\| & \leq c(1+j)^{-n / p}\left(\sum_{k \in \delta_{0}}\left|I_{j}\left(S_{j} f_{l}-f_{l}\right)\left(\frac{2 \pi k}{2 j+1}\right)\right| p\right)^{1 / p} \\
& \leq c(1+j)^{-n / p}\left(\sum_{k \in O_{\delta}}\left|\left(S_{j} f_{l}-f_{l}\right)\left(\frac{2 \pi k}{2 j+1}\right)\right|^{p}\right)^{1 / p}
\end{aligned}
$$

where $c$ is independent of $j, l$, and $f$. Next we pick out a sequence of meshes $\left\{M_{1}\right\}$ such that

$$
\left\{\left(\frac{2 \pi k}{2 j+1}\right): k \in Z^{n}\right\} \subset M_{1}=\left\{\left(\frac{2 \pi k}{2 M_{l+1}}\right): k \in Z^{n}\right\}(I=t-1, \ldots),
$$

where $(3 / 2) 2^{l-1} \leq M_{l} \leq c 2^{l}$ ( $c$ independent of $l$ and $t$ ) holds. According to $M_{l}$ we apply 
again (0.2). This leads to

$$
\left(\sum_{k \in O_{0}^{j}}\left|\left(S_{j} f_{l}-f_{l}\right)\left(\frac{2 \pi k}{2 j+1}\right)\right|\right)^{1 / p} \leq c 2^{\ln / p}\left\|S_{j} f_{l}-f_{l}\left|L_{P}\left\|\leq c 2^{\ln / p}\right\| f_{l}\right| L_{p}\right\| .
$$

Putting (2.7) into (2.6), summing up from $t-1$ to $\infty$ the desired inequality (2.3) follows

Remark 8: Using Remark 5 we can sharpen (2.4) a little bit. We have

$$
j^{M / P}\left\|f-I_{j} f \mid L_{p}\right\| \rightarrow 0 \text { if } j \rightarrow \infty
$$

for any $f \in B_{p, 1}^{n / P}$. In case $n=1$ this was observed first by K.I. Oskolkov [5].

Remark 9: In the one-dimensional case J. Prestin [7 - 10] has proved a result similar to $(2.8)$, but with $B_{p, 1}^{1 / P}\left(T^{1}\right)$ replaced by the set of functions with bounded variation.

As a consequence of Lemma 1 one obtains some estimates of the approximation error in $\|\cdot \mid C\|$.

Lemma 2: Let $p<\infty$ and $s>0$.

(i) For any $f \in B_{p, 1}^{n / p} \cup A_{\pi}$ there holds

$\left\|f-I_{j} f \mid C\right\| \rightarrow 0$ as $j \rightarrow \infty$.

(ii) There exists a constant c such that

$\sup _{j \in \mathbb{N}_{0}}(1+j)^{s}\left\|f-I_{j} f|C\|\leq c\| f| B_{p, \infty}^{s+n / P}\right\|$ for all $f \in B_{p, \infty}^{s+n / P}$.

(iii) There exists a constant $c$ such that

$$
\sup _{j \in \mathbb{N}_{0}}(1+j)^{s}(\log (1+j))^{-n}\left\|f-I_{j} f|C\|\leq c\| f| C^{s}\right\| \text { for all } f \in C^{s}=B_{\infty, \infty}^{s} \text {. }
$$

Proof: (i) Let $f \in A_{\pi}$. Then (2.9) follows from (2.2) since

$$
\left|f(x)-I_{j} f(x)\right| \leq \sum_{|m|>0} \sum_{k \in Q_{m}^{j}}|\hat{f}(k)| \text {. }
$$

Let $f \in B_{p, 1}^{n / p}$. Then we use the decomposition

$$
f-I_{j} f=f-V_{j / 2} f+l_{j}\left(V_{j / 2} f-f\right),
$$

with $V_{j / 2}$ defined in (1.7). From the embeddings $B_{p, 1}^{n / p} \hookrightarrow B_{\infty, 1}^{\circ} \hookrightarrow C$ (cf. H.-J. Schmeißer and $H$. Triebel [14]) and Remark 5 we know that

$$
\left\|f-V_{j / 2} f \mid C\right\| \rightarrow 0 \text { as } j \rightarrow \infty .
$$

Next we apply the Nikol'skij inequality (cf. H.-J. Schmeißer and H. Triebel [14]) and (2.3). This yields

$$
\left\|I_{j}\left(V_{j / 2} f-f\right)\left|C\left\|\leq c(1+j)^{n / P}\right\| I_{j}\left(V_{j / 2} f-f\right)\right| L_{p}\right\| \leq c\left\|f-V_{j / 2} f \mid B_{p, 1}^{n / P}\right\| .
$$

Using again Remark 5 we find

$$
\left\|I_{j}\left(V_{j / 2} f-f\right) \mid C\right\| \rightarrow \text { as } j \rightarrow \infty .
$$

Now, (2.13) and (2.14) complete the proof of (2.9).

(ii) We use the splitting stated in (2.12), Proposition 1,-and (2.3). This yields

$$
\left\|f-I_{j} f\left|C\|\leq\| f-V_{j / 2} f\right| C\right\|+c\left\|f-V_{j / 2} f\left|B_{p, 1}^{n / p}\left\|\leq c(1+j)^{s}\right\| f\right| B_{p, \infty}^{n / p+s}\right\| .
$$


For the last step we have used on the one hand the embedding $B_{p, \infty}^{n / p+s} \subset \rightarrow B_{\infty, \infty}^{s}=C^{s}$ and on the other hand Proposition 3 (see Section 3). This proves (2.10).

(iii) Since the first part of inequality (0.2) remains true if $p=1$ (cf. A. Zygmund [19]) we obtain

$$
\begin{aligned}
\left|I_{j} f(x)\right| & =\left(\frac{1}{2 j+1}\right)^{n}\left|\sum_{r \in O_{0}^{j}} \sum_{k \in O_{0}^{j}} f\left(x^{r}\right) \mathrm{e}^{i k(x-x)^{r}}\right| \\
& \leq \sup _{r \in O_{0}^{J}}\left|f\left(x^{r}\right)\right|\left(\frac{1}{2 j+1}\right)^{n} \sum_{r \in O_{\delta}^{j}}\left|\sum_{k \in O_{0}^{j}} \mathrm{e}^{\mathrm{i} k(x-x) r}\right| \\
& \leq c\left\|f\left|C\|\| \sum_{k \in O_{0}^{j}} \mathrm{e}^{i k x}\right| L_{1}\right\| \leq c(\log (1+j))^{n}\|f \mid C\| .
\end{aligned}
$$

Using this with $f-V_{j / 2} f$ instead of $f$, the desired inequality follows from (2.12) as in (ii)

The main result of this paper is formulated in the next

Theorem 1: Let $1<p<\infty$ and $s>n / p$.

(i) Let $0<q \leq \infty$. Then

$$
B_{p, q}^{s}=\left\{f \in C:|f(0)|+\left(\sum_{j=0}^{\infty}\left[(1+j)^{s-1 / q}\left\|f-I_{j} f \mid L_{p}\right\|\right]^{q}\right)^{1 / q}<\infty\right\}
$$

in the sense of equivalent quasi-norms.

(ii) Let $1<q<\infty$. Then

$$
F_{p, q}^{s}=\left\{f \in C:|f(0)|+\|\left(\sum_{j=0}^{\infty}\left[(1+j)^{s-1 / q}|f(x)|^{q}\right)^{1 / q} \mid L_{P} \|<\infty\right\}\right.
$$

in the sense of equivalent norms.

Proof: (i) Comparing the above characterization of $B_{p, q}^{s}$ with Proposition 1 it remains to prove that

$$
|f(0)|+\left(\sum_{j=0}^{\infty}\left[(1+j)^{s-1 / q}\left\|f-I_{j} f \mid L_{p}\right\|\right]^{q}\right)^{1 / q} \leq c\left\|f \mid B_{p, q}^{s}\right\|
$$

with $c$ independent of $f$. Let $0<q<\infty$. Again we use (2.3) and Proposition 1. This leads to

$$
\begin{aligned}
& \sum_{j=0}^{\infty}\left[(1+j)^{s-1 / q}\left\|f-I_{j} f \mid L_{p}\right\|\right]^{q} \\
& \quad \leq \sum_{j=0}^{\infty}\left[(1+j)^{s-1 / q}\left(\left\|f-S_{j} f\left|L_{p}\|+\| I_{j}\left(f-S_{j} f\right)\right| L_{p}\right\|\right)\right]^{q} \\
& \quad \leq c\left\|f \mid B_{p, q}^{s}\right\|^{q}+\sum_{j=0}^{\infty}\left[(1+j)^{s-1 / q}(1+j)^{-s / P}\left\|f-S_{j} f \mid B_{p, 1}^{n / p}\right\|\right]^{q} .
\end{aligned}
$$

We proceed with an estimate of the second term on the right-hand side of (2.16). Using

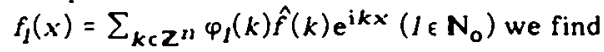

$$
\left\{\sum_{t=0}^{\infty} \sum_{j=2}^{2^{t+1}-2} 2^{t(s-n / p) q} 2^{-t}\left(\sum_{l=t-1}^{\infty} 2^{l n / p}\left\|\left(S_{j} f-f\right)_{l} \mid L_{p}\right\|\right)^{q}\right\}^{\min (1, q) / q}
$$




$$
\begin{aligned}
& s c \sum_{t=0}^{\infty}\left(\sum_{t=0}^{\infty} 2^{t(s-n / p) q} 2^{(l+t) q n / p}\left\|f_{l+t-1} \mid L_{p}\right\|^{q}\right)^{\min (1, q) / q} \\
& s c \sum_{t=0}^{\infty} 2^{-l(s-n / p) \min (1, q)}\left(\sum_{t=0}^{\infty} 2^{t s q}\left\|f_{t} \mid L_{p}\right\|^{q}\right)^{\min (1, q) / q} \\
& s c\left\|f \mid B_{p, q}^{s}\right\|^{\min (1, q)},
\end{aligned}
$$

since $s>n / p$ and

$$
\sup _{j}\left\|\left(S_{j} f\right)_{l}\left|L_{p}\left\|=\sup _{j}\right\| S_{j}\left(f_{l}\right)\right| L_{p}\right\| \leq c\left\|f_{l} \mid L_{p}\right\|
$$

( put $\varphi_{-1}=0$ ). Note that $L_{0} f=f(0)$. In view of this fact, Lemma 1 and $(2.16),(2.17)$ the desired inequality (2.15) follows if $q<\infty$. In case $q=\infty$ one has to modify the above considerations in an obvious way.

(ii) Using Proposition 2 the proof is reduced to establish the inequality

$$
\left|f(0) t+\left\|\left(\sum_{j=0}^{\infty}\left[(1+j)^{s-1 / q}\left|f(x)-l_{j} f(x)\right|\right]^{q}\right)^{1 / q}\left|L_{p}\|\leq c\| f\right| F_{p, q}^{s}\right\| .\right.
$$

Step 1: In order to prove (2.18) we consider at first the case $s>n$. Because of $F_{p, q}^{s} \subset \rightarrow$ $A_{\pi}$ (cf. Remark 7) we can apply (2.2). This yields

$$
f(x)-I_{j} f(x)=f(x)-S_{j} f(x)-\sum_{|m|>0}\left(\sum_{k \in Z^{n}} x_{m}^{j}(k) \hat{f}(k) \mathrm{e}^{\mathrm{i} k x}\right) \mathrm{e}^{-\mathrm{i} x m(2 j+1)},
$$

where $\chi_{m}^{j}$ is the characteristic function of $Q_{m}^{j}$. With the help of Proposition 1 a proof of $(2.18)$ is now reduced to a proof of

$$
\left\|\left(\sum_{j=0}^{\infty}\left[(1+j)^{s-1 / q}\left|\sum_{|m|>0}\left(\sum_{k \in Z^{n}} \chi_{m}^{j}(k) \hat{f}(k) \mathrm{e}^{\mathrm{i} k x}\right) \mathrm{e}^{-\mathrm{i} \times m(2 j+1)}\right|\right]^{q}\right)^{1 / q}\left|L_{p}\|\leq c\| f\right| F_{p_{1} q}^{s}\right\| .
$$

In order to obtain (2.19) we make use of Lizorkin's vector-valued Fourier-multiplier theorem for cubes with sides parallel to the axis (cf. H.-J. Schmeißer and H. Triebel [14]) and of

$$
Q_{m}^{j} \in K_{t+1}^{N_{0}, N_{1}} \text { if } 2^{t}-1 \leq j \leq 2^{t+1}-2,2^{l} \leq|m|<2^{l+1},
$$

where

$$
\begin{aligned}
& K_{0}^{N_{6}, N_{1}}=\left\{x:\left|x_{j}\right| \leq 2^{-N_{1}}(j=1, \ldots, n)\right\}, \\
& K_{t}^{N_{6}, N_{1}}=\left\{x:\left|x_{j}\right| \leq 2^{t+N_{0}}(j=1, \ldots, n)\right\} \backslash\left\{x:\left|x_{j}\right| \leq 2^{t-1-N_{1}}(j=1, \ldots, n)\right\}(t \in \mathbf{N})
\end{aligned}
$$

for appropriate $N_{0}, N_{1} \in N_{0}$. Let $\chi\left(K_{t}^{N_{6}, N_{1}}, \cdot\right)$ be the characteristic function of $K_{t}^{N_{0}, N_{1}}$. These yields

$$
\begin{aligned}
& \left\|\left(\sum_{r=0}^{\infty} 2^{t s q} \sum_{j=2^{t}-1}^{2^{t+1}-2} 2^{-t}\left|\sum_{|m|>0}\left(\sum_{k \in Z^{n}} \chi_{m}^{j}(k) \hat{f}(k) \mathrm{e}^{\mathrm{i} k x}\right) \mathrm{e}^{-\mathrm{i} x m(2 j+1)}\right|^{q}\right)^{t / q} \mid L_{p}\right\| \\
& \quad \leq \sum_{i=0}^{\infty} \sum_{2^{j} \leq|m|<2^{j+1}}\left\|\left(\sum_{t=0}^{\infty} 2^{t s q} \sum_{j=2^{2} 2^{t+1}-2} 2^{-t}\left|\sum_{k \in Z^{n}} \chi_{m}^{j}(k) \chi\left(K_{t+1}^{N, N_{1}}, k\right) \hat{f}(k) \mathrm{e}^{i k x}\right|^{q}\right)^{1 / q} \mid L_{p}\right\|
\end{aligned}
$$




$$
\begin{aligned}
& s c \sum_{l=0}^{\infty} 2^{l n}\left\|\left(\sum_{t=0}^{\infty} 2^{t s q}\left|\sum_{k \in Z^{n}} \chi\left(K_{t+1}^{N_{0}, N_{1}}, k\right) \hat{f}(k) e^{\mathrm{i} k x x}\right|^{q}\right)^{1 / q} \mid L_{p}\right\| \\
& \leq c \sum_{i=0}^{\infty} 2^{l(n-s)}\left\|f\left|F_{p, q}^{s}\|\leq c\| f\right| F_{p, q}^{s}\right\|,
\end{aligned}
$$

according to a so-called Lizorkin-type representation of $F_{p, q}^{s}$ (cf. H.-J. Schmeißer and $H$. Triebel [14]).

Step $2:$ We remove the restriction $s>n$. Note, that $B_{p, p}^{s}=F_{p, p}^{s}$. Furthermore, we have

$$
\begin{array}{lll}
{\left[F_{P_{0}, q_{0}}^{s_{1}}, F_{P_{1}}^{s_{1}} q_{1}\right]_{\theta}=F_{p, q}^{s}} & s=(1-\vartheta) s_{o}+\vartheta s_{1} \\
{\left[L_{P_{0}}(A), L_{p}(B)\right]_{\vartheta}=L_{p}\left([A, B]_{\vartheta}\right)} & \text { with } & \frac{1}{p}=\frac{1-\vartheta}{\rho_{0}}+\frac{\vartheta}{p_{1}} \\
{\left[l_{q_{0}}\left(A_{j}\right), l_{q_{1}}\left(B_{j}\right)\right]_{\vartheta}=I_{q}\left(\left[A_{j}, B_{j}\right]_{\vartheta}\right)} & & \frac{1}{q}=\frac{1-\vartheta}{q_{0}}+\frac{\vartheta}{q_{1}}
\end{array}
$$

(cf. Triebel [18]). We shall use (2.21) with $A_{j}=j^{s_{0}} \mathbb{C}, B_{j}=j^{s_{1}} \mathbb{C}$, and $A=I_{q_{0}}\left(A_{j}\right), B=I_{q_{1}}\left(B_{j}\right)$. Here $\mathbb{C}$ is the complex plane. Considering the linear operator $R: F_{p, q}^{s} \rightarrow L_{p}\left(I_{q}\left(j^{s-1 / q)} \mathbb{C}\right)\right.$ ), $R f=\left\{f-I_{j} f\right\}_{j=0}^{\infty}$ we know from the proof of (i) and from Step 2 that $R$ is bounded if $s>n / p$ and $p=q$ or $s>n$ and $1<p, q<\infty$. Hence, $R$ is bounded as a mapping with respect to the intermediate spaces $\left.R: F_{p, q}^{s} \rightarrow L_{p}\left(l_{q}\left(j^{s-1 / q}\right) \mathbb{C}\right)\right)(1<p, q<\infty ; s>n / p)$. That means, (2.18) is true also under these restrictions

Remark 10: The restriction $s>n / p$ in Theorem 1 seems to be natural. If $s<n / p$, then unbounded functions are contained in $B_{p, q}^{s}$ and hence, $I_{j} f$ makes no sense in general.

Remark 11: Parts of the assertions of Theorem 1 and of the Lemmas 1 and 2 are known if $n=1$. We refer to J. Prestin $[7,10]$ and K.I. Oskolkov [5]. Corresponding results in case of Whittaker's cardinal series are obtained in Sickel [16].

We are also interested in a characterization of function spaces if $p=\infty$. To this end we can employ an inequality due to Leindler [3]. Let $0<\mu<\infty$. Then

$$
\left\|\left(2^{-1} \sum_{j=2}^{2 l+1}\left|f(x)-S_{j} f(x)\right|^{\mu}\right)^{1 / \mu} \mid L_{\infty}\left(\mathrm{T}^{1}\right)\right\| \leq c E_{2^{j}}\left(f, C\left(\mathrm{~T}^{1}\right)\right),
$$

where $c$ is independent of $f$ and $l \in \mathbf{N}_{0}$. This implies

$$
\sup _{l \in \mathbb{N}_{0}} 2^{(1+1 / \mu) J}\left\|\left(2^{-1} \sum_{j=2}^{2 l+1}\left|f(x)-l_{j} f(x)\right|^{\mu}\right)^{1 / \mu}\left|C\left(\mathbf{T}^{1}\right)\|s c\| f\right| B_{\infty, 1}^{1+1 / \mu}\left(\mathrm{T}^{1}\right)\right\|
$$

and

$$
\sup _{l \in \mathbb{N}_{0}} 2^{s I}\left\|\left(2^{-1} \sum_{j=2}^{2 l+1}\left|f(x)-I_{j} f(x)\right|^{\mu}\right)^{1 / \mu}\left|C\left(\mathrm{~T}^{1}\right)\|\leq c\| f\right| C^{s}\left(\mathrm{~T}^{1}\right)\right\|
$$

if $I s \mu<\infty$ and $s>1+1 / \mu$. Extending (2.24) to $I_{q}$-norms one obtains a characterization of $B_{\infty, q}^{s}\left(\mathrm{~T}^{1}\right)$.

Theorem 2: Let $1 \leq \mu<\infty, 0<q \leq \infty$ and $s>1 / \min (1, q)+1 / \mu$. Then

$$
B_{\infty, q}^{s}\left(\mathbf{T}^{1}\right)=\left\{f \in C\left(\mathbf{T}^{i}\right):\left\|f \mid C\left(\dot{\mathbf{T}}^{1}\right)\right\|\right.
$$




$$
\left.+\left(\sum_{l=0}^{\infty} 2^{l s q}\left\|\left(2^{-1} \sum_{j=2}^{l+1} l^{1+1}\left|f(x)-I_{j} f(x)\right|^{\mu}\right)^{1 / \mu} \mid C\left(T^{1}\right)\right\| q\right)^{1 / q}<\infty\right\}
$$

in the sense of equivalent quasi-norms.

Remark 12: Assertions of this type with $I_{j} f$ replaced by $S_{j} f$ may be found in H.-J. Schmeißer and W. Sickel [13].

\section{Approximation in Besov and Sobolev norms}

In several papers the approximation order of $f-I_{j} f$ is studied in stronger norms than $\left\|\cdot \mid L_{p}\right\|$ (cf. R. Haverkamp [2], J. Prestin [7 - 10], S. Prößdorf and B. Silbermann [11]). The results derived in the preceding section can be generalized in a convenient way. The first step in doing this is the following characterization of Besov spaces (cf. A. Pietsch [6]).

Proposition 3: Let $1 \leq p \leq \infty, 0<q_{0}, q_{1} \leq \infty$, and $t, s>0$.

(i) We have

$$
B_{p, q_{0}}^{s+t}=\left\{f \in B_{p, q_{1}}^{t}:\left\|f \mid B_{p, q_{1}}^{t}\right\|+\left(\sum_{t=1}^{\infty}\left[j^{s-1 / q_{0}} E_{j}\left(f, B_{p, q_{1}}^{t}\right)\right]^{q_{0}}\right)^{1 / q_{0}}<\infty\right\}
$$

in the sense of equivalent quasi-norms.

(ii) If $1<p<\infty$, then $E_{j}\left(f, B_{p, q_{1}}^{t}\right)$ can be replaced by $\left\|f-S_{j} f \mid B_{p, q_{1}}^{t}\right\|$ in (i).

As a consequence of this proposition and Theorem 1 we obtain the following

Theorem 3: Let $1<p<\infty, 0<q_{0}, q_{1} \leq \infty, t \geq 0$ and $s>0$. Let additionally $s+t>n / p$. Then we have

$$
B_{p, q_{0}}^{s+t}=\left\{f \in B_{p, q_{1}}^{t}:\left\|f \mid B_{p, q_{1}}^{t}\right\|+\left(\sum_{j=0}^{\infty}\left[(1+j)^{s-1 / q_{0}}\left\|f-I_{j} f \mid B_{p, q_{1}}^{t}\right\|\right]^{q_{0}}\right)^{1 / q_{0}}<\infty\right\}
$$

in the sense of equivalent quasi-norms.

Proof: By Proposition 3 it is sufficient to prove

$$
\left\|f\left|B_{p, q_{1}}^{t}\left\|+\left(\sum_{j=0}^{\infty}\left[(1+j)^{s-1 / q_{0}}\left\|f-I_{j} f \mid B_{p, q_{1}}^{t}\right\|\right]^{q_{0}}\right)^{1 / q_{0}} \leq c\right\| f\right| B_{p, q_{0}}^{s+t}\right\|
$$

for some constant $c$, independent of $f$. Therefore, we use the splitting from (2.5). Again by applying Proposition 3 it suffices to consider the term $I_{j}\left(S_{j} f-f\right)$. Let $2^{v} \leq j<2^{v+1}$. Then (2.3) implies

$$
\begin{gathered}
\left\|J_{j}\left(S_{j} f-f\right) \mid B_{p, q_{1}}^{t}\right\| \leq\left(\sum_{i=0}^{v+1} 2^{I t q_{1}}\left\|\sum_{k \in Z^{n}} \varphi_{j}(k) I_{j}\left(S_{j} f-f\right)(k) e^{i k x} \mid L_{p}\right\|^{q_{2} \mid 1 / q_{1}}\right. \\
\quad s c(1+j)^{t}\left\|I_{j}\left(S_{j} f-f\right)\right\| L_{p} \| \leq c(1+j)^{t}\left(\left\|f-S_{j} f\left|L_{p}\|+\| f-I_{j} f\right| L_{p}\right\|\right) .
\end{gathered}
$$

This leads to

$$
\left(\sum_{j=0}^{\infty}\left[(1+j)^{s-1 / q_{0}}\left\|f-I_{j} f \mid B_{p, q_{1}}^{t}\right\|\right]^{q_{0}}\right)^{1 / q_{0}}
$$




$$
\leq c\left(\left\|f \mid B_{p, q_{0}}^{s+t}\right\|+\left(\sum_{j=0}^{\infty}\left[(1+j)^{s+t}\left(\left\|f-S_{j} f\left|L_{p}\|+\| f-S_{j} f\right| L_{p}\right\|\right)\right]^{q_{0}}\right)^{1 / q_{0}}\right) \leq c\left\|f \mid B_{p, q_{0}}^{s+t}\right\|
$$

since $s+t>n / p$ ensures that Theorem 1 can be applied $D$

Remark 13: As a consequence of embeddings for Besov-Triebel-Lizorkin spaces on the $n$-torus one obtains characterizations of $B_{p, q}^{s}$ via approximation by Lagrange interpolating polynomials in certain norms. For instance, by $B_{p, 1}^{0} c \rightarrow L_{p} \subset \rightarrow B_{p, \infty}^{0}$ one obtains Theorem 1 as an application of Theorem 3 . Furthermore, by $B_{p, 1}^{t} \subset \rightarrow W_{p}^{t} \subset \rightarrow B_{p, \infty}^{t}(t \in \mathbb{N})$ one can replace $B_{p, q_{1}}^{t}$ in (3.1) by the Sobolev spaces $W_{p}^{t}$. This improves some results of $S$. Prößdorf and B. Silbermann [11] and J. Prestin $[7,10]$.

\section{REFERENCES}

[1] BUTZER, P.L.: A survey of the Whittaker-Shannon sampling theoren and some of its extensions. J. Math. Res. Exp. 3 (1983), $185-212$.

[2] HAVERKAMP, R: Approximationsgute der Ableitungen bei trigonometrischer Interpolation. Math. 2. 179 (1982), 59 - 67.

(3) LEINDLER, L.: On summability of Fourier series. Acta Sci. Math. (Szeged) 29 (1968), $147-162$

[4] LIZORKIN, P.I., and D.G. ORLOVSKIJ.: Some interpolation formulas for trigonometric and exponential polynomials and estinates connected with them. (Russian.) Trudy Sem. Sobolev Math. Inst. Nkad. Nauk SSSR, Sib. Otd. 1 (1976), 60 - 71.

[5] OSKOLKOV, K.I.: Inequalities of the "large sieve" type and applications to trigono metric approximation. Anal. Math. 12 (1986), 143 - 166.

[6] PIETSCH, A.: Approximation spaces. J. Approx. Theory 32 (1981), 115 - 134.

[7] PRESTIN, J:: Approximation in perjodischen Lipschitz-Raumen. Thesis. Rostock: Wilhelm-Pieck-Universität 1985.

[8] PRESTIN, J.: Trigonometrische Approximation von Funktionen beschränkter Variation. Math. Nachr. 122 (1985), $29-43$.

[9] PRESTIN, J.: Trigonometric interpolation of functions of bounded variation. In: Constructive Theory of Functions. Proc. Int. Conf. Varna 1984. Sofia : Publ. House Bulg. Acad. Sci. 1984, 699- 703.

[10] PRESTIN, J.: On the approximation by de la Vallee' Poussin means and interpolatory polynomials in Lipschitz norms. Anal. Math. 13 (1987), 251 - 259.

[11] PRÖSSDORF, S., and B. SILBERMANN.: Projektionsverfahren und die naherungsweise Lösung singularer Gleichungen (Teubner-Texte zur Mathematik: Vol. 12). Leipzig: B.G. Teubner Verlagsges. 1977.

[12] SCHMEISSER, H.-J., and W. SICKEL: On strong summability of multiple Fourier series and smoothness properties of functions. Anal. Math. 8 (1982), $57-70$.

[13] SCHMEISSER, H.-J., and W. SICKEL: On strong summability of multiple Fourier series and approximation of periodic functions. Math. Nachr. 133 (1987), 211 - 236.

[14] SCHMEISSER, H.-J., and H. TRIEBEL.: Topics in Fourier Analysis and Function Spaces. Leipzig: Akad. Verlagsges. Geest \& Portig 1986, and Chichester: Wiley 1986.

[15] SICKEL, W.: Periodische Räume: Maximalungleichungen. Fouriersche Multiplikatoren und Beziehungen zur starken Summierbarkeit mehrdimensionaler trigonometrischer Reihen. Thesis. Jena: Friedrich-Schiller-Universität 1984.

[16] SICKEL, W.: Characterization of Besov-Triebel-Lizorkin spaces via approximation by Whittaker's cardinal series. Preprint. Jena: Friedrich-Schiller-Univ. 1990, 1 - 20.

[17] H.TRIEBEL : Fourier Analysis and Function Spaces (Teubner-Texte zur Mathematik: Vo1. 7). Leipzig: B.G. Teubner Verlagsges. 1977.

[18] H.TRIEBEL: Interpolation Theory. Function Spaces, Differential Operators. Am-

37 Analysis, Bd. 10. Heft 4 (1991) 
sterdam-New York-Oxford: North-Holland Publ. Comp. 1978, and Berlin: De. Verlag Wiss. 1978.

[19] ZYGMUND, A.: Trigonometric Series, Vo1. I and II. Sec. ed. Cambridge: Cambr. Univ. Press 1977.

Received 13.02.1990; in revised form 30.01.1991

Dr. Winfried Sickel

Institut fur Mathematik der Friedrich-Schiller-Universität

Universitats-Hochhaus, 17. OG

D (Ost) - 6900 Jena 